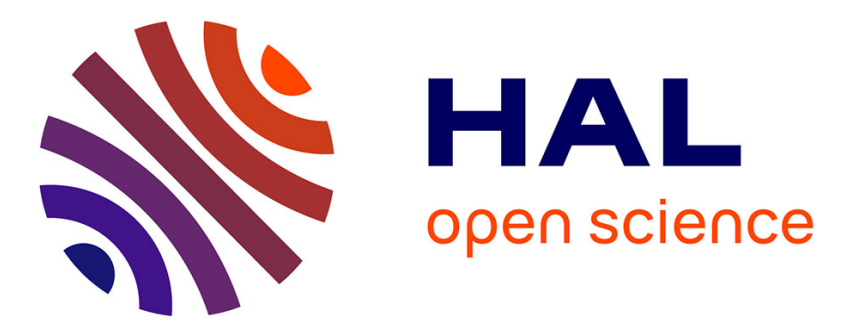

\title{
Bounded Nash type controls for uncertain linear systems
} Marc Jungers, Eugênio Castelan, Edson Roberto de Pieri, Hisham

Abou-Kandil

\section{To cite this version:}

Marc Jungers, Eugênio Castelan, Edson Roberto de Pieri, Hisham Abou-Kandil. Bounded Nash type controls for uncertain linear systems. Automatica, 2008, 44 (7), pp.1874-1879. 10.1016/j.automatica.2007.10.035 . hal-00197153

\section{HAL Id: hal-00197153 https://hal.science/hal-00197153}

Submitted on 15 Jul 2013

HAL is a multi-disciplinary open access archive for the deposit and dissemination of scientific research documents, whether they are published or not. The documents may come from teaching and research institutions in France or abroad, or from public or private research centers.
L'archive ouverte pluridisciplinaire HAL, est destinée au dépôt et à la diffusion de documents scientifiques de niveau recherche, publiés ou non, émanant des établissements d'enseignement et de recherche français ou étrangers, des laboratoires publics ou privés. 


\title{
Bounded Nash type controls for uncertain linear systems *
}

\author{
Marc Jungers a ${ }^{\text {, Eugênio B. Castelan }}{ }^{\mathrm{c}}$, Edson R. De Pieri ${ }^{\mathrm{c}}$, Hisham Abou-Kandil ${ }^{\mathrm{b}}$ \\ ${ }^{\mathrm{a}}$ CRAN UMR 7039 CNRS - Nancy Université ENSEM, \\ 2 avenue de la foret de Haye \\ F-54516 Vandoeuvre cedex, France. \\ ${ }^{\mathrm{b}}$ SATIE, ENS Cachan, CNRS, UniverSud \\ 61 av du President Wilson, \\ F-94230 Cachan, France. \\ ${ }^{\mathrm{c}}$ Federal University of Santa Catarina \\ DAS/CTC/UFSC 88049900 Florianopolis SC Brazil.
}

\begin{abstract}
This paper deals with multicriteria controls for systems coping with polytopic uncertainties. The proposed controls are inspired by a Nash strategy for exactly known systems, reformulated as a nonconvex coupling between Semi-Definite Programming problems. The extension to the uncertain case duplicates the Linear Matrix Inequalities for all vertices of the polytope. A new iterative algorithm using Semi-Definite Programming is provided to design bounded Nash type controls for uncertain systems. A numerical example is given to illustrate the design method.
\end{abstract}

Key words: Nash strategies, Game theory, Algebraic Riccati equations, Linear matrix inequalities (LMI), Robustness, Polytopic uncertainty.

\section{Introduction}

For systems governed by several controllers (decision makers or players) where each controller aims to minimize its own cost function, Nash strategy offers a nice framework to study control robustness. In fact, such a strategy has an inherent robustness property since no player can improve his/her payoff by deviating unilaterally from his/her Nash strategy once the equilibrium is attained. Several approaches are proposed using Nash strategy to design robust controls for linear systems $[14,8]$. A way to treat uncertainty is to interpret perturbation as an exogenous input (a fictitious player) $[7,19]$. In [19], the definition of equilibria is ex-

\footnotetext{
* This paper was not presented at any IFAC meeting. Corresponding author M. Jungers. Tel. +33 -3-83-59-57-04. Fax +33 -3-83-59-56-44.

Email addresses: Marc. Jungers@cran.uhp-nancy.fr (Marc Jungers), eugenio@das.ufsc.br (Eugênio B. Castelan), edson@das.ufsc.br (Edson R. De Pieri), abou-kandil@satie.ens-cachan.fr (Hisham Abou-Kandil).

1 This work was partially supported by the program CAPES/COFECUB n ${ }^{\circ}$ 489/05 and by CNPq, Brazil.
}

tended to deal with two cases: a soft-constrained formulation, inspired by [4], where the fictitious player is introduced in the criteria via a weighting matrix, and a hard-constrained formulation where the fictitious input is of bounded energy.

An extended definition of Nash equilibrium is proposed in [18]. For a bounded energy disturbance, the cost value is deviating from the nominal one by a distance which increases when the disturbance energy grows up [15]. In addition, if the disturbance is periodic and of known period, using a learning lapse of time, it is possible to estimate the effect of the disturbance and a pure Nash equilibrium is obtained. However, the associated equations are not easy to solve and the characteristics of the disturbance should be known.

The common feature of all these techniques is that only unstructured uncertainty is dealt with (perturbations or neglected dynamics) while the system's model is supposed to be well known. For linear-quadratic zero-sum games with structured uncertainties, sufficient conditions are provided in [2] to guarantee a bound for the cost function of each player. Finding such a bound is based on the existence of a solution for an appropriately 
parameterized Riccati equation.

The purpose of this paper is to design robust controls inspired by Nash strategy for systems exhibiting parameters uncertainties in their models. The main difficulty here is to minimize the deviation from the equilibrium point, thus guaranteeing an upper bound for the payoffs. For differential games, sufficient conditions for obtaining a Nash equilibrium are well known $[5,17,16,1]$. However, variational calculus or dynamic programming are not well adapted to deal with parameters uncertainties in the dynamic model.

The outline of the paper is as follows: in section 2, the general setting for a Nash equilibrium with a closed loop information structure is recalled for linear-quadratic games with exactly known dynamics. It is shown that obtaining the Nash control is equivalent to solving a non-convex optimization problem. In section 3, Linear Matrix Inequalities (LMI) are used to reformulate the problem of determining Nash controls as a sequence of decoupled convex optimization problems. This new formulation allows to design robust controls inspired by Nash equilibrium in the case of uncertain polytopic systems (section 4). A numerical algorithm is given in section 5 based on iterative solution of Semi-Definite Programming (SDP) problems. An upper bound for the cost functions is derived and the properties of the proposed solution are discussed in section 6 . An example is treated in section 7 to illustrate the obtained theoretical results.

\section{Nash Strategy for certain system}

Consider a two-players linear-quadratic differential nonzero-sum game, on an infinite time horizon:

$$
\left\{\begin{array}{l}
\dot{x}(t)=A x(t)+B_{1} u_{1}(t)+B_{2} u_{2}(t), \\
x\left(t_{0}\right)=x_{0},
\end{array}\right.
$$

where $x(t) \in \mathbb{R}^{n}, u_{i} \in \mathcal{L}^{2}\left(\left[t_{0},+\infty\right), \mathbb{R}^{r_{i}}\right)(i, j \in\{1,2\}$ verifying $i \neq j$ and $\left.n, r_{i} \in \mathbb{N}^{*}\right)$ and with the costs functionals :

$$
J_{i}=\int_{t_{0}}^{+\infty}\left(x^{T} Q_{i} x+u_{1}^{T} R_{i 1} u_{1}+u_{2}^{T} R_{i 2} u_{2}\right) \mathrm{d} \tau .
$$

All weighting matrices are constant and symmetric with $Q_{i}>0, R_{i j}=D_{i j}^{T} D_{i j} \geq 0$ and $R_{i i}=D_{i}^{T} D_{i}>0$.

The pair $\left(u_{1}^{*}, u_{2}^{*}\right)$ corresponds to a Nash equilibrium $[5,11,12]$ if the following relations are satisfied for each admissible controls $^{2}\left(u_{1}, u_{2}\right)$ :

$$
\left\{\begin{array}{l}
J_{1}\left(u_{1}, u_{2}^{*}\right) \geq J_{1}\left(u_{1}^{*}, u_{2}^{*}\right), \\
J_{2}\left(u_{1}^{*}, u_{2}\right) \geq J_{2}\left(u_{1}^{*}, u_{2}^{*}\right) .
\end{array}\right.
$$

Thus, at Nash equilibrium with $\left(u_{1}^{*}, u_{2}^{*}\right)$, the player who chooses to change his/her strategy cannot improve his/her payoff.

If there exist positive definite matrices $\left(P_{1}, P_{2}\right)$, satisfying the Coupled Algebraic Riccati type Equations (CARE)

$$
0_{n}=\mathcal{N}_{1}\left(P_{1}, P_{2}\right)=\mathcal{N}_{2}\left(P_{1}, P_{2}\right),
$$

where

$$
\begin{aligned}
\mathcal{N}_{1}\left(P_{1}, P_{2}\right)= & A^{T} P_{1}+P_{1} A+Q_{1}+P_{2} S_{12} P_{2} \\
& -P_{2} S_{2} P_{1}-P_{1} S_{2} P_{2}-P_{1} S_{1} P_{1} \\
\mathcal{N}_{2}\left(P_{1}, P_{2}\right)= & A^{T} P_{2}+P_{2} A+Q_{2}+P_{1} S_{21} P_{1} \\
& -P_{1} S_{1} P_{2}-P_{2} S_{1} P_{1}-P_{2} S_{2} P_{2}
\end{aligned}
$$

(with $S_{i j}=B_{j} R_{j j}^{-1} R_{i j} R_{j j}^{-1} B_{j}^{T}$, and $S_{i}=B_{i} R_{i i}^{-1} B_{i}^{T}$ ), then the controls

$$
u_{i}^{*}(t)=-R_{i i}^{-1} B_{i}^{T} P_{i} x(t),
$$

constitute a set of Nash equilibrium strategies within the class of admissible control functions mentioned in footnote 2 if the game has a closed-loop information structure. So, in particular, the matrix $\left(A-S_{1} P_{1}-S_{2} P_{2}\right)$ is stable. The proof is similar as the one shown in the theorem 6 . In this case, the criteria associated with the equilibrium verify

$$
J_{i}\left(u_{1}^{*}, u_{2}^{*}\right)=x_{0}^{T} P_{i} x_{0} .
$$

Equations (4) are hard to solve due to the presence of quadratic coupling terms between $P_{1}$ and $P_{2}$. To the best of our knowledge there is no explicit conditions guaranteeing the existence of solutions for CARE (4). Implicit conditions and special cases are provided in [9] and [10]. Only numerical algorithms without proof of convergence, are available to solve these equations $[10,13]$.

\section{Formulation of CARE via coupled nonconvex optimization problems}

This section provides a reformulation of sufficient conditions for a Nash strategy with closed-loop information

\footnotetext{
2 A couple of admissible controls is defined as a couple of controls which stabilizes the system (1) and allows the existence of both finite criteria $J_{1}$ and $J_{2}$.
} 
structure, given by (7), whenever positive definite solutions $P_{1}$ and $P_{2}$ can be found for CARE (4). This new formulation is particularly interesting to deal with uncertain systems, as will be shown later.

Theorem 1 Assume that the coupled nonconvex optimization problems $\Pi_{1}$ and $\Pi_{2}$ below admit a solution $\left(E_{1}, W_{1}, Y_{1}\right)$ and $\left(E_{2}, W_{2}, Y_{2}\right)$, then

$$
\left\{\begin{array}{l}
\mathcal{N}_{1}\left(E_{1}, E_{2}\right) \leq 0_{n} \\
\mathcal{N}_{2}\left(E_{1}, E_{2}\right) \leq 0_{n}
\end{array}\right.
$$

are satisfied. In addition if $\left(A+B_{j} K_{j}, B_{i}, C_{z_{i}}\right)$, where $K_{j}$ is defined by (16), are minimal, then the equalities of (9) are achieved, that is (4) are solved.

$\Pi_{1}$ : Minimize $\operatorname{Tr}\left(E_{1}\right)$, with respect to $\left(E_{1}, W_{1}, Y_{1}\right)$, subject to:

$$
\begin{aligned}
& W_{1}>0_{n}, \\
& {\left[\begin{array}{cc}
E_{1} & I_{n} \\
I_{n} & W_{1}
\end{array}\right] \geq 0_{2 n},} \\
& \mathcal{M}_{\left(A, B_{1}, B_{2}\right)}^{1,2}\left(Y_{1}, W_{1}, K_{2}\right) \leq 0_{2 n+r_{1}},
\end{aligned}
$$

$\Pi_{2}$ : Minimize $\operatorname{Tr}\left(E_{2}\right)$, with respect to $\left(E_{2}, W_{2}, Y_{2}\right)$, subject to:

$$
\begin{aligned}
& W_{2}>0_{n}, \\
& {\left[\begin{array}{cc}
E_{2} & I_{n} \\
I_{n} & W_{2}
\end{array}\right] \geq 0_{2 n},} \\
& \mathcal{M}_{\left(A, B_{1}, B_{2}\right)}^{2,1}\left(Y_{2}, W_{2}, K_{1}\right) \leq 0_{2 n+r_{2}},
\end{aligned}
$$

under the coupling conditions

$$
K_{1}=Y_{1} W_{1}^{-1}, \quad K_{2}=Y_{2} W_{2}^{-1},
$$

with $K_{1}$ and $K_{2}$ corresponding to the feedback controls of the two players. The matrices in LMI (12) and (15) are defined by

$$
\begin{aligned}
& \mathcal{M}_{\left(A, B_{1}, B_{2}\right)}^{i, j}\left(Y_{i}, W_{i}, K_{j}\right)=
\end{aligned}
$$

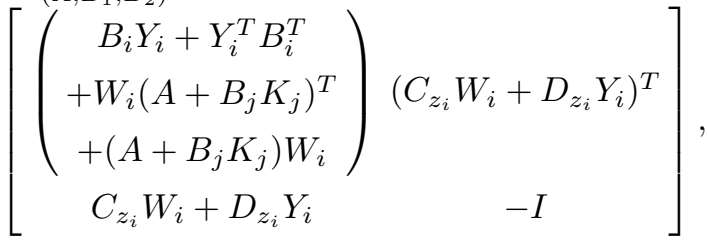

where

$$
C_{z_{i}}=\left[\begin{array}{c}
\left(Q_{i}+K_{j}^{T} R_{i j} K_{j}\right)^{1 / 2} \\
0_{r_{i} \times n}
\end{array}\right], \quad D_{z_{i}}=\left[\begin{array}{c}
0_{n \times r_{i}} \\
D_{i}
\end{array}\right],
$$

Proof Since both nonconvex optimization problems in Theorem 1 are symmetric, only the proof of the first one, corresponding to the first inequality in (3) is proposed. By using the Schur Complement on the LMI (12), we obtain

$$
\begin{aligned}
& W_{1}\left(A-B_{2} K_{2}\right)^{T}+\left(A-B_{2} K_{2}\right) W_{1}+B_{1} Y_{1}+Y_{1}^{T} B_{1}^{T} \\
& \quad+\left(C_{z_{1}} W_{1}+D_{z_{1}} Y_{1}\right)^{T}\left(C_{z_{1}} W_{1}+D_{z_{1}} Y_{1}\right) \leq 0_{n} .
\end{aligned}
$$

A simple completion-of-squares argument [6, p.115] [3] allows to show that

$$
Y_{1}=-\left(D_{z_{1}}^{T} D_{z_{1}}\right)^{-1} B_{1}^{T}=-R_{11}^{-1} B_{1}^{T} .
$$

In the same way, we obtain for $\Pi_{2}$ :

$$
Y_{2}=-\left(D_{z_{2}}^{T} D_{z_{2}}\right)^{-1} B_{2}^{T}=-R_{22}^{-1} B_{2}^{T} .
$$

Injecting these two last expressions and the coupling conditions (16) into (19), inequalities (9) are satisfied.

By fixing $K_{j}$ in problem $\Pi_{i}$, the obtained LMI can be rearranged as a standard Riccati equation for the system $\left(A+B_{j} K_{j}, K_{i}, C_{z_{i}}\right)$. If this system is minimal, then according to $[6$, p. 114$]$, the bound of inequalities $(9)$ is achieved.

\section{Remark 2 By noting}

$$
z_{1}=C_{z_{1}} x+D_{z_{1}} u_{1}
$$

an other proof can be provided by interpreting the first inequality in (3) as the energy of the fictitious output $z_{1}$ of the system when the control $u_{2}=K_{2} x$ is applied [6, section 7.4.1]. We obtain in this case that this energy does not exceed

$$
x_{0}^{T} W_{1}^{-1} x_{0},
$$

under the constraints (10) and (12). This bound is achieved for a state-feedback $u_{1}=K_{1} x$, where $K_{1}=Y_{1} W_{1}^{-1}$.

Remark 3 As for the solution of the CARE (4), the solution of the coupled nonconvex optimization problems $\Pi_{1}$ and $\Pi_{2}$ is not necessarily unique and numerically difficult. However, a Semi-Definite Programming (SDP) version of the iterative algorithm in $[1, p$. 340] can be obtained from Theorem 1 by subsequently solving $\Pi_{1}$ and $\Pi_{2}$ with fixed values of $K_{2}$ and $K_{1}$, respectively. Although no proof of convergence exists for this iterative procedure, 
numerical experiments have shown the ability to numerically solve the CARE or equivalently, the coupled nonconvex optimization problems $\Pi_{1}$ and $\Pi_{2}$ iteratively. This kind of numerical procedure can be particularly adapted to obtain a Nash control strategy in the case of uncertain polytopic systems, as shown in the next sections.

\section{Guaranteed costs for uncertain linear systems}

Consider an uncertain linear dynamical system described by a $N$-polytopic model. The system (1) becomes

$$
\left\{\begin{array}{l}
\dot{x}(t)=A(\lambda) x(t)+B_{1}(\lambda) u_{1}(t)+B_{2}(\lambda) u_{2}(t), \\
x\left(t_{0}\right)=x_{0} .
\end{array}\right.
$$

The initial state $x_{0}$ is assumed to be known. The vector $\lambda=\left(\lambda_{1}, \cdots, \lambda_{N}\right)$ corresponds to the unknown parameters of the system:

$$
\begin{aligned}
& {\left[A(\lambda) B_{1}(\lambda) B_{2}(\lambda)\right] \in} \\
& \operatorname{Co}\left\{\left[\begin{array}{lll}
A_{1} & B_{1,1} & B_{2,1}
\end{array}\right], \cdots,\left[\begin{array}{lll}
A_{N} & B_{1, N} & B_{2, N}
\end{array}\right]\right\} \text {, }
\end{aligned}
$$

where Co denotes the convex hull of the $N$ vertices. We note then

$$
\left[A(\lambda) B_{1}(\lambda) B_{2}(\lambda)\right]=\sum_{k=1}^{N} \lambda_{k}\left[\begin{array}{lll}
A_{k} & B_{1, k} & B_{2, k}
\end{array}\right],
$$

with $\lambda \in \Lambda=$

$$
\left\{\lambda \mid \lambda_{k} \in[0,1], \forall k \in\{1, \cdots, N\}, \sum_{k=1}^{N} \lambda_{k}=1\right\} .
$$

All weighting matrices in the criteria $J_{i}(i=1,2)$, defined by (2), are exactly known. We extend the notation $J_{i}\left(u_{1}, u_{2}\right)$ to $J_{i}\left(u_{1}, u_{2}, \lambda\right)$, when the parameter $\lambda$ is applied to the system (24).

We propose to provide here a design for a couple of controls $\left(\tilde{u}_{1}^{*}, \tilde{u}_{2}^{*}\right)$ inspired by the SDP formulation of Nash equilibrium. These controls, which are independent of $\lambda$ lead to a guaranteed level of criteria $J_{1}$ and $J_{2}$ for polytopic uncertainties, with arbitrary evolution.

Remark 4 Except in the particular case of a single vertex polytope, $\left(\tilde{u}_{1}^{*}, \tilde{u}_{2}^{*}\right)$ may not be associated with a Nash equilibrium.

\section{Numerical Algorithm}

The iterative procedure for solving problems $\Pi_{1}$ and $\Pi_{2}$, summarized before in the case of precisely known system, is now adapted for treating the case of uncertain systems. The basic idea consists of subsequently solving each problem $\Pi_{i}$, repeating inequalities (12) and (15) for each vertex of polytope Co $\left(A_{k}, B_{1, k}, B_{2, k}\right)$. To determine the controls $\tilde{u}_{1}^{*}$ and $\tilde{u}_{2}^{*}$, the SDP $\Pi_{1}$ and $\Pi_{2}$ are solved alternately under the constraints (12) and (15) for all vertices and $K_{1}$ or $K_{2}$ is updated with the coupling conditions (16). The algorithm is as follows ${ }^{3}$ :

Initialization: Set $K_{2}=0_{r_{2} \times n} .{ }^{4}$

Step 1: Solve the SDP

$\tilde{\Pi}_{1}$ : Minimize $\operatorname{Tr}\left(E_{1}\right)$, with respect to $\left(E_{1}, W_{1}, Y_{1}\right)$, subject to:

$$
\begin{aligned}
& W_{1}>0_{n}, \\
& {\left[\begin{array}{cc}
E_{1} & I_{n} \\
I_{n} & W_{1}
\end{array}\right] \geq 0_{2 n},} \\
& \left\{\begin{array}{c}
\mathcal{M}_{\left(A_{1}, B_{1,1}, B_{2,1}\right)}^{1,2}\left(Y_{1}, W_{1}, K_{2}\right) \leq 0_{2 n+r_{1}}, \\
\vdots \\
\mathcal{M}_{\left(A_{N}, B_{1, N}, B_{2, N}\right)}^{1,2}\left(Y_{1}, W_{1}, K_{2}\right) \leq 0_{2 n+r_{1}} .
\end{array}\right.
\end{aligned}
$$

Compute $K_{1}=Y_{1} W_{1}^{-1}$.

Step 2: Solve the SDP

$\tilde{\Pi}_{2}$ : Minimize $\operatorname{Tr}\left(E_{2}\right)$, with respect to $\left(E_{2}, W_{2}, Y_{2}\right)$, subject to:

$$
\begin{aligned}
& W_{2}>0_{n} \\
& {\left[\begin{array}{cc}
E_{2} & I_{n} \\
I_{n} & W_{2}
\end{array}\right] \geq 0_{2 n},} \\
& \left\{\begin{array}{c}
\mathcal{M}_{\left(A_{1}, B_{1,1}, B_{2,1}\right)}^{2,1}\left(Y_{2}, W_{2}, K_{1}\right) \leq 0_{2 n+r_{2}}, \\
\vdots \\
\mathcal{M}_{\left(A_{N}, B_{1, N}, B_{2, N}\right)}^{2,1}\left(Y_{2}, W_{2}, K_{1}\right) \leq 0_{2 n+r_{2}} .
\end{array}\right.
\end{aligned}
$$

Compute $K_{2}=Y_{2} W_{2}^{-1}$.

Step 3: Repeat Steps 1 and 2, until all LMI (28-33) are simultaneously satisfied.

When the algorithm stops, the last variables are noted $Y_{i}^{*}, E_{i}^{*}=\left(W_{i}^{*}\right)^{-1}$ and $K_{i}^{*}$. The controls $\tilde{u}_{1}^{*}$ and $\tilde{u}_{2}^{*}$ are given by $\tilde{u}_{i}^{*}(t)=K_{i}^{*} x(t)$.

Remark 5 For a system without uncertainty (only one vertex), the algorithm is a set of coupled optimization problems where at each step the solution of a single Riccati equation is computed. This simplified algorithm is

\footnotetext{
3 As usual in the literature, we note not strict inequalities, however the strict inequalities are used by the LMI solver

4 Other initial values for $K_{2}$ may be considered for initializing the algorithm, the obtained solution being dependent on the choosen initial value. The choice $K_{2}=0_{r_{2} \times n}$ means that, in the first iteration $\tilde{\Pi}_{1}$ solves a standard LQR problem.
} 
a Semi-Definite Programming version of a classical algorithm [1, p. 340]. To the best of our knowledge, there exists no condition which guarantees that the algorithm stops.

\section{Properties of solution}

Theorem 6 (Stability) When the controls $\tilde{u}_{1}^{*}$ and $\tilde{u}_{2}^{*}$ are applied, the evolution matrix

$$
A^{*}(\lambda)=A(\lambda)+B_{1}(\lambda) K_{1}^{*}+B_{2}(\lambda) K_{2}^{*}
$$

is Hurwitz for any $\lambda \in \Lambda$. Furthermore, the corresponding closed-loop control system is stable, even if the uncertainty parameter vector is arbitrary time-variant: $\lambda=\lambda(t) \in \Lambda, \forall t \geq 0$.

Proof By rearranging the inequalities (30) and using Schur complement, we obtain the Lyapunov inequality

$$
\begin{aligned}
& \left(A^{*}(\lambda)\right)^{T} E_{i}^{*}+E_{i}^{*}\left(A^{*}(\lambda)\right) \\
& \leq-Q_{i}-\left(K_{i}^{*}\right)^{T} R_{i i} K_{i}^{*}-\left(K_{j}^{*}\right)^{T} R_{i j} K_{j}^{*}<0 .
\end{aligned}
$$

The result ensues from the positive definiteness of $E_{i}^{*}$ and $Q_{i}$. Even if $\lambda$ varies, the associated Lyapunov's function implies the quadratic stability of the system $[6$, section 5.1]. Theorem 6 guarantees also that $J_{1}$ and $J_{2}$ are finite for every vector $\lambda$ by applying the controls $\tilde{u}_{i}^{*}(t)=K_{i}^{*} x(t)$.

Theorem 7 (Upper Bound of Criteria) When the controls $\tilde{u}_{i}^{*}(t)=K_{i}^{*} x(t)$ are applied on the system depending on $\lambda$,

$$
J_{i}\left(\tilde{u}_{1}^{*}, \tilde{u}_{2}^{*}, \lambda\right)=x_{0}^{T} G_{i}(\lambda) x_{0} \leq x_{0}^{T} E_{i}^{*} x_{0},
$$

where $G_{i}(\lambda)$ is given by the solving of the Lyapunov equation

$$
\begin{aligned}
& A^{*}(\lambda)^{T} G_{i}(\lambda)+G_{i}(\lambda) A^{*}(\lambda)= \\
& \quad-\left(Q_{i}+\left(K_{i}^{*}\right)^{T} R_{i i} K_{i}^{*}+\left(K_{j}^{*}\right)^{T} R_{i j} K_{j}^{*}\right) .
\end{aligned}
$$

\section{Proof}

Injecting the equation (37) into (35), we obtain

$A^{*}(\lambda)^{T}\left(\left(W_{i}^{*}\right)^{-1}-G_{i}(\lambda)\right)+\left(\left(W_{i}^{*}\right)^{-1}-G_{i}(\lambda)\right) A^{*}(\lambda) \leq 0$.

Since $A^{*}(\lambda)$ is stable, we have $\left(W_{i}^{*}\right)^{-1} \geq G_{i}(\lambda)$, which ensures $x_{0}^{T}\left(W_{i}^{*}\right)^{-1} x_{0}=x_{0}^{T} E_{i}^{*} x_{0} \geq x_{0}^{T} G_{i}(\lambda) x_{0}$.

\section{$7 \quad$ Example}

In this section, we consider an extension of an example given in [1, p. 341] including a polytopic model composed of three vertices.

$$
\begin{aligned}
A_{1} & =\left(\begin{array}{rrrr}
-0.086 & 0.027 & 0.018 & -0.455 \\
-0.051 & -1.510 & -0.047 & -4.020 \\
0.100 & -0.214 & -0.757 & 1.322 \\
0 & 0 & 1.000 & 0
\end{array}\right), \\
A_{2} & =\left(\begin{array}{rrrr}
0.013 & 0.027 & 0.018 & -0.455 \\
0.148 & -0.510 & 0.052 & -4.020 \\
0.100 & 0.785 & -0.657 & 1.322 \\
0 & 0 & 1.000 & 0
\end{array}\right), \\
A_{3} & =\left(\begin{array}{rrrr}
-0.036 & 0.127 & 0.018 & -0.455 \\
0.058 & -1.000 & 1.002 & -4.020 \\
0.100 & 0.485 & -0.607 & 1.422 \\
0 & 0 & 1.000 & 0
\end{array}\right),
\end{aligned}
$$

$$
\begin{aligned}
& B_{1,1}=\left(\begin{array}{llll}
0.342 & 2.844 & -5.020 & 0
\end{array}\right)^{T}, \\
& B_{1,2}=\left(\begin{array}{llll}
0.542 & 3.244 & -6.020 & 0
\end{array}\right)^{T} \text {, } \\
& B_{1,3}=\left(\begin{array}{llll}
4.442 & 1.044 & -4.520 & 0
\end{array}\right)^{T} \text {, } \\
& B_{2,1}=\left(\begin{array}{llll}
0.176 & -7.692 & 5.290 & 0
\end{array}\right)^{T} \text {, } \\
& B_{2,2}=\left(\begin{array}{llll}
0.176 & -7.492 & 4.690 & 0
\end{array}\right)^{T} \text {, } \\
& B_{2,3}=\left(\begin{array}{llll}
0.376 & -7.592 & 4.890 & 0
\end{array}\right)^{T} \text {. }
\end{aligned}
$$

The weighting matrices are given by

$$
\begin{gathered}
Q_{1}=\operatorname{diag}(3.5 ; 2 ; 4 ; 5), Q_{2}=\operatorname{diag}(1.5 ; 6 ; 3 ; 1), \\
R_{11}=1 ; R_{12}=0.25 ; R_{21}=0.6 ; R_{22}=2, \\
x_{0}=\left(\begin{array}{llll}
1 & 0 & 1 & 1
\end{array}\right)^{T} .
\end{gathered}
$$

The algorithm stops after 17 steps, for this example. We obtain

$$
\begin{aligned}
x_{0}^{T} E_{1}^{*} x_{0} & =20.3306, \quad x_{0}^{T} E_{2}^{*} x_{0}=11.7189, \\
K_{1}^{*} & =\left(\begin{array}{llll}
-0.2193 & 0.0401 & 0.1730 & 0.2574
\end{array}\right), \\
K_{2}^{*} & =\left(\begin{array}{llll}
0.9143 & 2.2963 & -0.8192 & -2.3623
\end{array}\right) .
\end{aligned}
$$


The performance of the controls $\left(\tilde{u}_{1}^{*}, \tilde{u}_{2}^{*}\right)$ is shown in Fig. 1 and Fig. 2. The figures depict two surfaces corresponding to $J_{i}\left(\tilde{u}_{1}^{*}, \tilde{u}_{2}^{*}, \lambda\right)=x_{0}^{T} G_{i}(\lambda) x_{0}$ and the upper bound $x_{0}^{T} E_{i}^{*} x_{0}$, for each $\lambda \in \Lambda$.

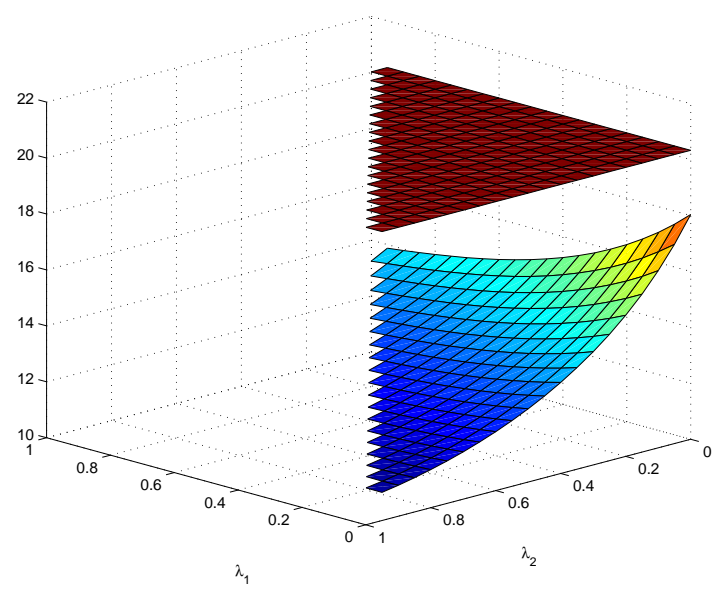

Fig. 1. Performance on $J_{1}\left(\tilde{u}_{1}^{*}, \tilde{u}_{2}^{*}, \lambda\right)$.

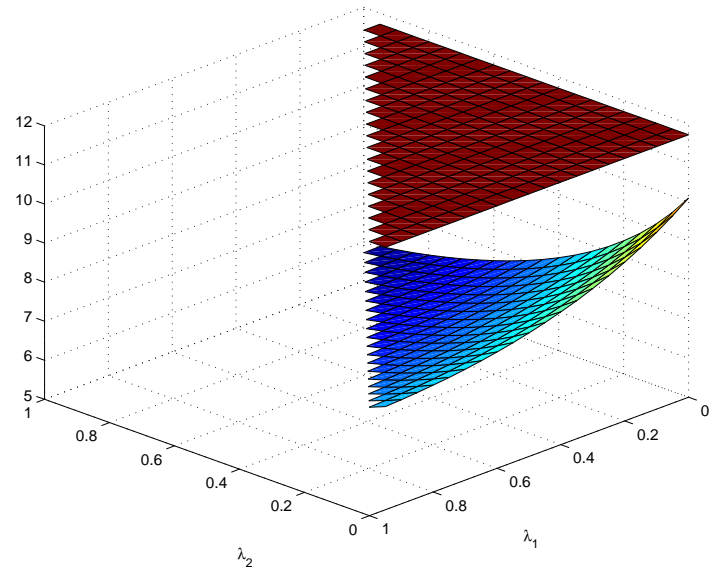

Fig. 2. Performance on $J_{2}\left(\tilde{u}_{1}^{*}, \tilde{u}_{2}^{*}, \lambda\right)$.

\section{Conclusion}

Control design inspired by Nash equilibrium for systems with a polytopic representation of uncertainty is proposed. To determine such robust controls for polytopic uncertain systems, a reformulation of the nonconvex optimization problem associated with Nash strategy is provided. Such a formulation allows the introduction of parameters uncertainty in the system's model, by decomposing the nonconvex optimization problem into a sequence of convex ones. SDP leads to an efficient algorithm to compute robust controls, inspired by Nash strategies.

\section{References}

[1] H. Abou-Kandil, G. Freiling, V. Ionescu, and G. Jank. Matrix Riccati Equations in Control and Systems Theory. Birkhäuser, 2003.

[2] F. Amato, M. Mattei, and A. Pironti. Guaranteeing cost strategies for linear quadratic differential games under uncertain dynamics. Automatica, 38:507-515, 2002.

[3] V. Balakrishnan and L. Vandenberghe. Semidefinite programming duality and linear time-invariant systems. IEEE Transactions on Automatic Control, 48(1):30-41, 2003.

[4] T. Başar and P. Bernhard. $\mathcal{H}_{\infty}$-Optimal Control and Related Minimax Design Problems: A Dynamic Game Approach. Birkhäuser, 1995.

[5] T. Başar and G. J. Olsder. Dynamic Noncooperative Game Theory. SIAM, 1995.

[6] S. Boyd, L. El Ghaoui, E. Feron, and V. Balakrishnan. Linear Matrix Inequalities in System and Control Theory. SIAM Studies in Applied Mathematics, 1994.

[7] H. Chen, C.W. Scherer, and F. Allgöwer. A game theoretic approach to nonlinear robust receding horizon control of constrained systems. volume 5, pages 3073-3077, Albuquerque, New Mexico, June 1997. Proceedings of the American Control Conference.

[8] X. Chen and K. Zhou. Multiobjective $\mathcal{H}_{2} / \mathcal{H}_{\infty}$ control design. SIAM Journal Control Optimization, 40(2):628-660, 2001.

[9] J. C. Engwerda. LQ Dynamic Optimization and Differential Games. Wiley, 2005.

[10] G. Freiling, G. Jank, and H. Abou-Kandil. On global existence of solutions to coupled matrix Riccati equations in closed-loop Nash games. IEEE Transactions on Automatic Control, 41(2):264-296, February 1996.

[11] Y. C. Ho. Survey paper: Differential games, dynamic optimization and generalized control theory. Journal of Optimization Theory and Applications, 6(3):179-209, 1970.

[12] Y. C. Ho, A. E. Bryson, and S. Baron. Differential games and optimal pursuit-evasion strategies. IEEE Transactions on Automatic Control, AC-10(4):385-389, 1965.

[13] M. Jungers, E. De Pieri, and H. Abou-Kandil. Solving coupled algebraic Riccati equations from closed-loop Nash strategy, by lack of trust approach. In IFAC-CAO, Cachan, France, 26-28 Avril 2006.

[14] D.J.N. Limebeer, B.D.O. Anderson, and H. Hendel. A Nash game approach to mixed $\mathcal{H}_{2} / \mathcal{H}_{\infty}$ control. IEEE Transactions on Automatic Control, 39(1):69-82, January 1994.

[15] M. Jimenez Lizarraga and A. Poznyak. $\epsilon$-equilibrium in LQ differential games with bounded uncertain disturbances. In $13^{\text {th }}$ IFAC Workshop on Control Applications of Optimisation, Cachan, France, 26-28 April 2006.

[16] A. W. Starr and Y. C. Ho. Further properties of nonzerosum differential games. Journal of Optimization Theory and Applications, 3(4):207-219, 1969.

[17] A. W. Starr and Y. C. Ho. Nonzero-sum differential games. Journal of Optimization Theory and Applications, 3(3):184206, 1969.

[18] K. Tanaka and K. Yokoyama. On $\epsilon$-equilibrium point in a noncooperative N-person game. Journal of Mathematical Analysis and Applications, 160:413-423, 1991.

[19] W.A. van den Broek, J.C. Engwerda, and J.M. Schumacher. Robust equilibria in indefinite linear-quadratic differential games. Journal of Optimization Theory and Applications, 119(3):565-595, 2003. 